\title{
Recent progress in fungus-derived bioactive agents for targeting of signaling machinery in cancer cells
}

This article was published in the following Dove Press journal:

Drug Design, Development and Therapy

26 March 2015

Number of times this article has been viewed

\author{
Xiukun Lin' \\ Ammad Ahmad Farooqi ${ }^{2}$ \\ Muhammad Ismail ${ }^{2}$ \\ 'Department of Pharmacology, Capital \\ Medical University, Beijing, People's \\ Republic of China; ${ }^{2}$ Institute of \\ Biomedical and Genetic Engineering, \\ Islamabad, Pakistan
}

\begin{abstract}
It is becoming increasingly understood that tumor cells may have different mutations and dependencies on diverse intracellular signaling cascades for survival or metastatic potential. Overexpression of oncogenes, inactivation of tumor suppressor genes, genetic/epigenetic mutations, genomic instability, and loss of apoptotic cell death are some of the mechanisms that have been widely investigated in molecular oncology. We partition this multicomponent review into the most recent evidence on the anticancer activity of fungal substances obtained from in vitro and xenografted models, and these fungal substances modulate expression of oncogenic and tumor suppressor miRNAs. There are some outstanding questions regarding fungus-derived chemical-induced modulation of intracellular signaling networks in different cancer cell lines and preclinical models. Certain hints have emerged, emphasizing mechanisms via which apoptosis can be restored in TRAIL-resistant cancer cells. Reconceptualization of the knowledge obtained from these emerging areas of research will enable us to potentially identify natural agents with notable anticancer activity and minimal off-target effects. Integration of experimentally verified evidence obtained from cancer cell line gene expression with large-scale functional screening results and pharmacological sensitivity data will be helpful in identification of therapeutics with substantial efficacy. New tools and technologies will further deepen our understanding of the signaling networks that underlie the development of cancer, metastasis, and resistance to different therapeutics at both a personal and systems-wide level.
\end{abstract}

Keywords: fungal products, cell signaling, cancer, apoptosis, miRNA, xenograft

\section{Introduction}

High-throughput technologies have revolutionized our current understanding of the genomically complex nature of cancer. Phenomenal breakthroughs have been made in the field of molecular oncology, as evidenced by advancements in genetics, genomics, and proteomics. With overwhelmingly expanding information about cancer, it is now more understandable that overexpression of oncogenes, inactivation of tumor suppressor genes, genetic/epigenetic mutations, genomic instability, dysregulation of spatiotemporally controlled intracellular signaling cascades, and loss of apoptotic cell death are some of the mechanisms that underlie the development and progression of cancer. Rapidly accumulating experimentally verified data are deepening our knowledge about wide-ranging mechanisms that induce resistance against different therapeutic agents. It is noteworthy that bioactive ingredients isolated from natural sources are now widely appreciated.

\section{Anticancer activity of bioactive fungal components: in vitro and in vivo evidence}

The polysaccharide fraction purified from a culture broth of Ganoderma formosanum has been shown to have notable anticancer activity. When administered intraperitoneally
Correspondence: Ammad Ahmad Farooqi Institute of Biomedical and Genetic Engineering (IBGE), KRL Hospital, Mauve Area, G-9/I, Islamabad, Pakistan Emailammadahmad638@yahoo.com 
or orally, the polysaccharide fraction efficiently inhibited tumor growth in xenografted mice. It is noteworthy that the growth inhibitory effects exerted by the polysaccharide fraction were impaired in severe combined immunodeficiency (SCID) mice. CD4 ${ }^{+} \mathrm{T}$-cells and $\mathrm{CD}^{+} \mathrm{T}$-cells were noted to be essential for the polysaccharide fraction-mediated tumor growth-retarding effects. ${ }^{1}$

\section{Wnt signaling cascade}

Wnt-induced intracellular signaling is an extensively studied mechanism reported to underpin the development and progression of cancer. $\beta$-catenin, a well-known downstream effector, is reported to be modulated by positive and negative regulators, and phosphorylated $\beta$-catenin is degraded via the ubiquitin pathway. Different regulators have been noted to control $\beta$-catenin, including axin, adenomatous polyposis coli, casein kinase, and glycogen synthase kinase (GSK)-3 $\beta$. Wnt-induced intracellular signaling sequesters $\beta$-catenin from GSK-3 $\beta$ and promotes its accumulation in the nucleus, where $\beta$-catenin works synchronously with T-cell factor/ lymphocyte enhancer binding factor to stimulate expression of target genes. Cordycepin isolated from Cordyceps sinensis, a parasitic fungus, has been shown to decrease protein levels as well as nuclear accumulation of $\beta$-catenin. Cordycepin exerted its biological effects by enhancing GSK-3 $\beta$-mediated phosphorylation of $\beta$-catenin, and these effects were impaired in cells treated with GSK-3 $\beta$ inhibitors. Moreover, phosphatidylinositol-4 5-bisphosphate 3-kinase (PI3K)/Akt-mediated inactivation of GSK-3 $\beta$ was also inhibited, as evidenced by a decrease in phospho-Akt levels. ${ }^{2}$ Protein-bound polysaccharide isolated from Phellinus linteus also efficiently reduced $\beta$-catenin and its target gene in SW480 colon cancer cells overexpressing $\beta$-catenin (Figure 1). Protein-bound polysaccharide inhibited T-cell factor/lymphocyte enhancer binding factor reporter activity in SW480 cells transiently transfected with a luciferase reporter construct. ${ }^{3}$ Destruxin B isolated from Metarhizium anisopliae has also been found to inhibit the Wnt signaling pathway. $\beta$-catenin/Tcf4 transcriptional activity was also marked inhibited in destruxin B-treated HCT116 and SW480 cells ${ }^{4}$ (Figure 1). Antrodia camphorata grows on germinated brown rice and recently it has been reported that ethanolic extract is effective against HT-29 colon cancer cells. Ethanolic extract was further characterized and ethyl acetate-mediated anticancer activity was more pronounced. The $\beta$-catenin pathway was notably inhibited in cancer cells treated with ethyl acetate. ${ }^{5}$

\section{TGF signaling}

A confluence of information indicates that fungal lactones, ie, (S)-curvularin, oxacyclododecindione, dehydrocurvularin,

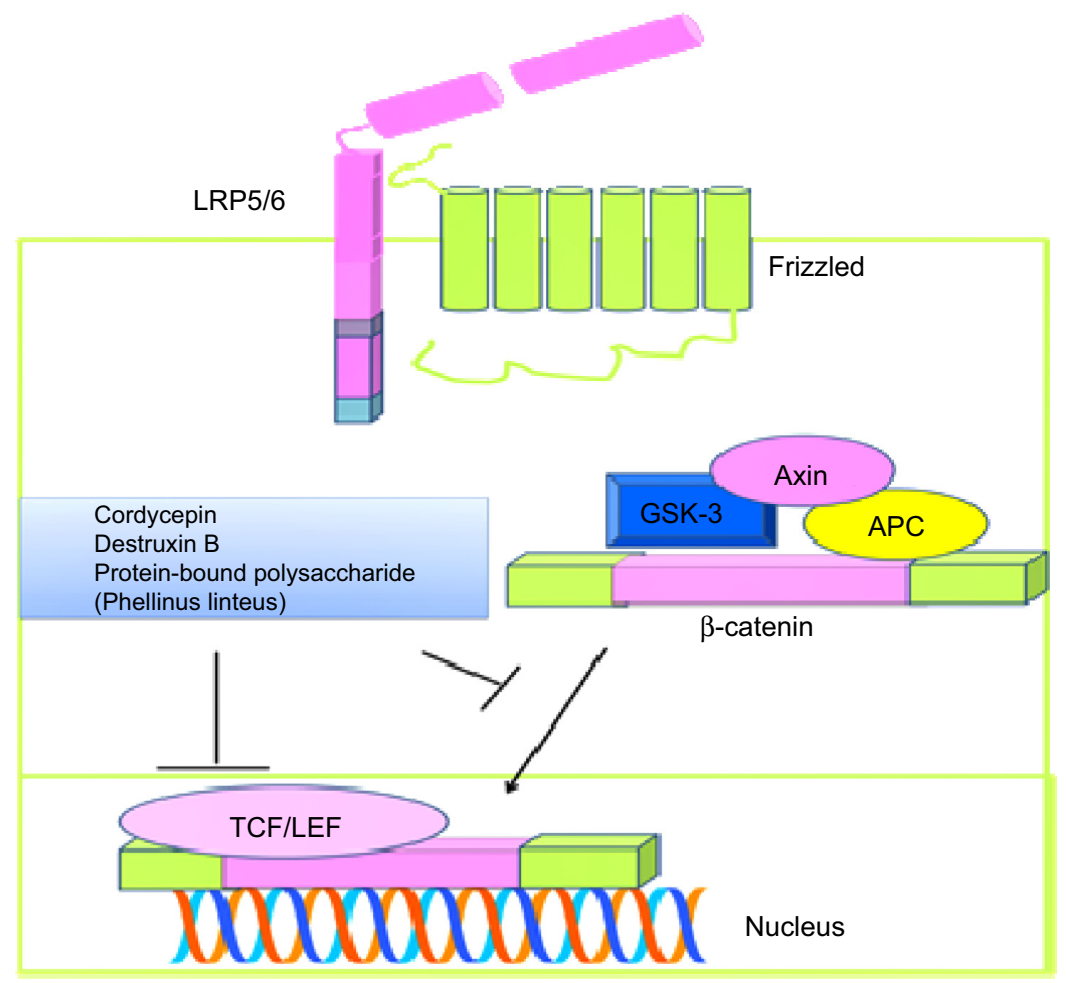

Figure I Wnt-induced intracellular signaling and fungal product-mediated inhibition of nuclear accumulation of $\beta$-catenin and transcriptional upregulation of target genes. 
and galiellalactone, considerably inhibit transforming growth factor (TGF)-induced intracellular signaling, and SMAD2/ 3-mediated stimulation of the transcriptional network was also reduced in MDA-MB-231 and HepG2 cells (Figure 2). ${ }^{6}$ Circumstantial evidence also indicates that TGF-induced intracellular activation of SMAD2/3 is dramatically reduced in MDA-MB-231 and HepG2 cells treated with trichodimerol (a fungal metabolite). ${ }^{7}$ Cordycepin isolated from Cordyceps militaris effectively reduced production of IL-2 and TGF- $\beta$ in mice xenografted with cancer cells. ${ }^{8}$

\section{NF-KB signaling}

Nuclear factor kappa B (NF- $\mathrm{BB}, \mathrm{p} 65$ and p50) is cytosolic and coexists with its inhibitor, I $\kappa \mathrm{B}$ kinase. Trichothecin, a metabolite isolated from an endophytic fungus, is a potent inhibitor of trichothecin and its target genes. Detailed mechanistic insights indicated that inhibition of I $\kappa \mathrm{B}$ kinase-mediated phosphorylation of I $\mathrm{I} \mathrm{B} \alpha$ facilitated sequestration and nuclear accumulation of $\mathrm{p} 65$. NF- $\kappa \mathrm{B}$ triggered expression of XIAP, cyclin D1, and Bcl-xL. However, treatment with trichothecin dramatically reduced
I $\kappa \mathrm{B} \alpha$ phosphorylation and nuclear accumulation of NF- $\kappa \mathrm{B}$. Enforced expression of constitutively active I $\kappa$ Binase- $\beta$ considerably reduced trichothecin-induced apoptosis. ${ }^{9}$ Western blot analysis confirmed a decrease in IкB kinase, p50, and p65 protein levels in fumigaclavine-treated MCF-7 cells. ${ }^{10}$ Destruxin B isolated from M. anisopliae inhibited I $\kappa \mathrm{B}$ kinase- $\alpha$ and NF- $\kappa \mathrm{B}$, and interestingly the I $\mathrm{BB} \alpha$ level was enhanced. ${ }^{4}$

\section{PI3K/Akt signaling pathway}

Phosphoinositide-dependent protein kinase 1 (PDK1) is a serine/threonine kinase reported to modulate wide-ranging downstream effectors, particularly PI3K and Akt. It is now known that ligand-binding sites present in the PDK1 kinase domain can be targeted using different natural agents. Neoalbaconol, a novel molecule isolated from the Albatrellus confluens fungus, has been shown to dock into the PDK1 adenosine triphosphate-binding pocket, thus forming hydrogen bonds with the PDK1 backbone. This inhibition abrogated PDK1-mediated levels of phospho-Akt at the 308th and 473rd serine residues in C666-1 nasopharyngeal carcinoma cells. ${ }^{11}$

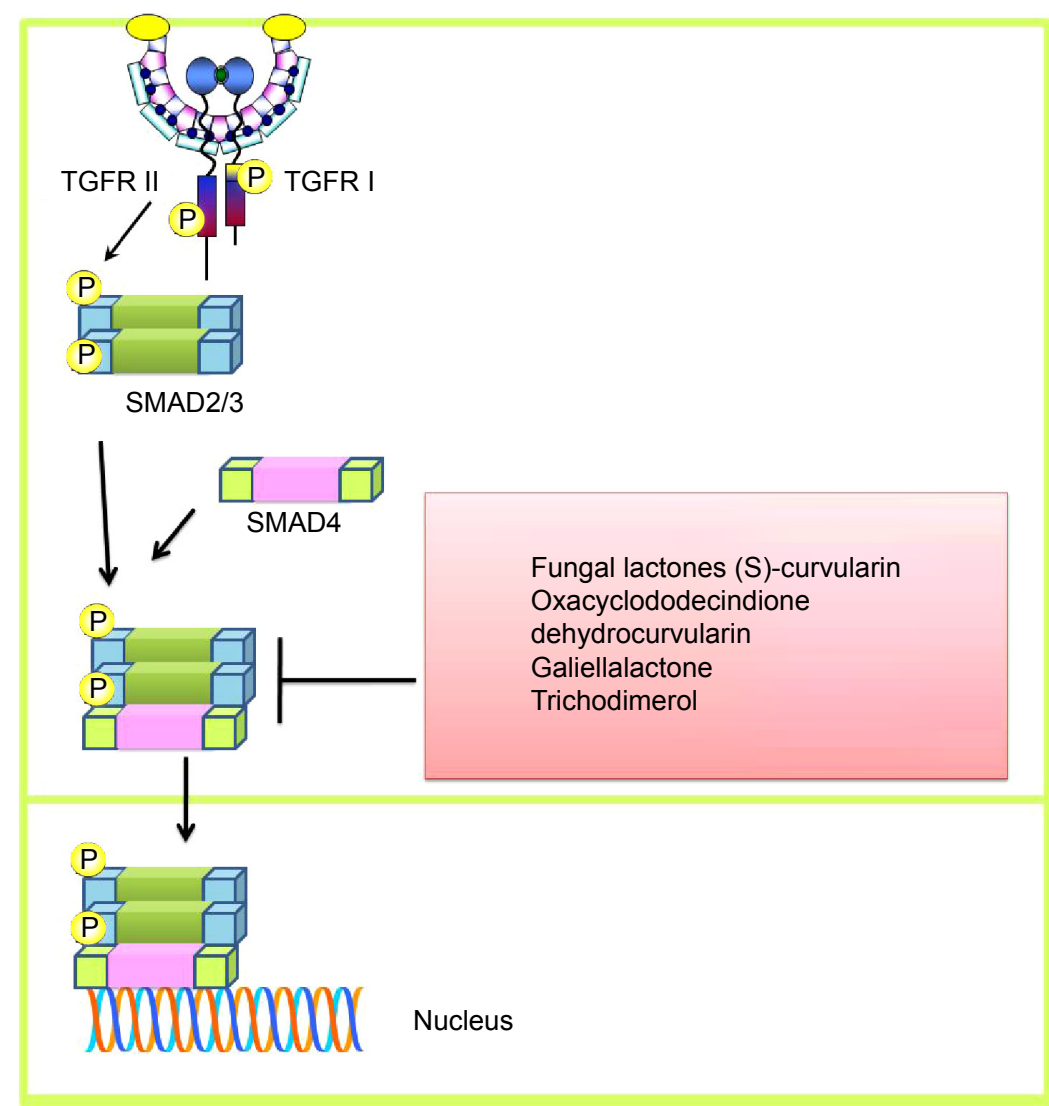

Figure 2 Transforming growth factor-induced intracellular signaling and fungal product-mediated inhibition of nuclear accumulation of SMAD and transcriptional upregulation of target genes.

Abbreviation: TGFR, transforming growth factor receptor. 
PI3K-mediated phosphorylation of Akt was also inhibited in fumigaclavine-treated MCF-7 cells. ${ }^{10}$ Destruxin B isolated from $M$. anisopliae inhibited the PI3K/Akt pathway by reducing Akt expression. ${ }^{4}$ Extract of Pleurotus pulmonarius also inhibited phosphor-Akt levels, and enforced expression of constitutively active Akt impaired the extract-mediated inhibitory effects on Huh7 and Hep3B liver cancer cells. Moreover, sensitivity to cisplatin was enhanced in cancer cells treated with the extract. ${ }^{12}$ Ochratoxin, a secondary metabolite of Penicillium, has recently been noted to potentiate carcinogenesis. It was shown mechanistically that ochratoxin-treated cells displayed active PI3K/Akt and MEK/ERK1-2 intracellular signaling cascades. It was further shown that c-Met, a cell surface receptor, was also functionally active upon treatment of cells with ochratoxin. ${ }^{13}$ There is a recent report suggesting that sterigmatocystin in combination with ochratoxin or citrinin worked with effective synergy against Hep3B hepatocellular cancer cells. ${ }^{14}$

\section{MMPs: novel targets of fungal products}

Contemporary studies indicated that 3-O-methylfunicone dramatically reduced $\alpha \mathrm{V} \beta 5$ integrin and matrix metalloproteinase (MMP)-2 expression by exerting inhibitory effects on ERK-mediated signaling in human mesothelioma cells. ${ }^{15}$ Fumigaclavine C, a chemical isolated from Aspergillus fumigatus, also efficiently targeted MMPs. MMP-2 and MMP-9 protein expression was remarkably reduced in fumigaclavinetreated MCF-7 cells. The decrease in MMPs reduced the migratory and invasive potential of MCF-7 cells. ERK, JNK, and p38 mitogen-activated protein kinase (MAPK) are also targeted by fumigaclavine, as evidenced by reduced phosphoERK, JNK, and p38 MAPK levels in fumigaclavine-treated MCF-7 cells. ${ }^{10}$ Protein-bound polysaccharide isolated from $P$. linteus and destruxin B isolated from $M$. anisopliae exerted inhibitory effects on MMP-2 and MMP-9 activity in HT1080 and SW480 colon cancer cells. ${ }^{3,4}$ Cordycepin and zhankuic acid, isolated from Antrodia cinnamomea, considerably enhanced tissue inhibitors of MMP (TIMP-1 and TIMP-2) in CL1-0 lung adenocarcinoma cells. ${ }^{16}$

\section{Cyclins and cyclin-dependent kinases}

Mitogenic signals promote heterodimerization of cyclin D1 and cyclin E1 with cyclin-dependent kinase (CDK)-4 and CDK-2, respectively. These phosphorylate the Rb proteins, causing release of E2Fs. Cyclin E is also a target gene of E2F that later complexes with CDK-2 to phosphorylate $\mathrm{Rb}$. Fumigaclavine-treated MCF-7 breast cancer cells displayed a marked decrease in CDK-2, CDK-4, and cyclin E protein expression levels. ${ }^{10}$ Moreover, the fungus-derived compound cephalochromin downregulated cyclin D1, cyclin E, CDK-2, and CDK-4 in A549 lung cancer cells, thus abrogating the association of cyclins with their respective CDKs. ${ }^{17}$

\section{Targeting of negative regulators of death signaling}

Paxilline, isolated from Penicillium paxilli, has been shown to effectively induce apoptosis in TRAIL-resistant glioma cell lines. Paxilline-treated glioma cells displayed a marked increase in the TRAIL receptor, DR5. Interestingly, paxilline markedly reduced cellular levels of FLICE-inhibitory protein and survivin by potentiating proteasome-triggered degradation. ${ }^{18}$ Fungus-derived cephalochromin effectively reduced $\mathrm{Bcl}-\mathrm{xL}$ and survivin expression and activated caspase-3 via caspase- 8 and caspase-9 in A549 lung cancer cells. ${ }^{17}$ Caspase-8-mediated proteolytically cleaved truncated Bid and Bax were also elevated in DU145 prostate cancer cells treated with glionitrin A, isolated from A. fumigatus. ${ }^{19}$

Verrucarin A, isolated from Myrothecium verrucaria, is a protein synthesis inhibitor reported to activate caspase-3 and upregulate Bax in MDA-MB-231 and T47D breast cancer cells. Moreover, Bcl-2 expression was notably reduced in verrucarin-treated breast cancer cells. ${ }^{20}$ The ratio of Bax to Bcl-2 was also enhanced in human laryngeal carcinoma cells treated with polysaccharides of Lepista sordida. Release of cytochrome c was noted in Hep-2 cells along with a marked increase in caspase- 9 activity. ${ }^{21}$

\section{Fungal products awake "guardian angels"}

Polysaccharides isolated from Ganoderma atrum considerably enhanced cell surface expression of Toll-like receptor-4 in macrophages and induced intracellular signaling. Moreover, there was evident nuclear accumulation of the p65 subunit of NF- $\kappa \mathrm{B}$ and degradation of $\mathrm{I} \kappa \mathrm{B} \alpha$. Further, phosphorylated levels of p38-MAPK, ERK-1/2, and c-JNK were also increased in macrophages. ${ }^{22}$ In another study, a similar mode of action was demonstrated, as polysaccharides did not kill CT26 colorectal carcinoma cells directly but exerted inhibitory effects on proliferation of CT26 cells by activating peritoneal macrophages. Tumor growth was also remarkably inhibited in mice with CT26 xenografts. ${ }^{23}$

\section{mTOR signaling}

Extract of Ganoderma lucidum efficiently decreased levels of phospho-mTOR (mammalian target of rapamycin) and 
its downstream effectors, including p70S6K, S6, p-S6, and p-4E-BP1, in SUM-149 inflammatory breast cancer cells. ${ }^{24}$ Monascuspiloin efficiently induced apoptosis in LNCaP prostate cancer cells by inhibiting PI3K/Akt/mTORinduced downstream signaling. ${ }^{25}$ Antrocin isolated from A. camphorata decreased phospho-Akt and phospho-mTOR levels in MDA-MB-231 cells. ${ }^{26}$

\section{Targeting of Rho GDP dissociation inhibitor}

RHO-specific guanine nucleotide dissociation inhibitors (RHoGDIs) have emerged as versatile regulators of RHO proteins and are reported to protect RHO proteins from degradation. It has previously been convincingly demonstrated that secalonic acid isolated from Penicillium spp. considerably enhanced caspase-3-mediated cleavage of RhoGDI 2. Secalonic acid-treated HL60 cells displayed a marked increase in the cleaved product of RhoGDI 2. ${ }^{27}$ Alcohol extracts of $A$. camphorata mycelia also downregulated RhoGDI- $\alpha$ expression in A549 lung carcinoma cells. ${ }^{28}$

\section{VEGF-induced signaling}

Vascular endothelial growth factor (VEGF) is a proangiogenic molecule for vascular endothelial cells and a potent vascular permeability enhancer. Mounting evidence indicates that VEGF has a key role during angiogenesis, proliferation, and migration of endothelial cells. Toluquinol exerted inhibitory effects on VEGF-induced and fibroblast growth factor-induced intracellular signaling. Interestingly, it has been suggested that capillary tube formation of endothelial cells on Matrigel is substantially reduced upon treatment with toluquinol, a methylhydroquinone isolated from a marine fungus. Additionally, the invasive, migratory and proteolytic activities of endothelial cells were also suppressed. ${ }^{29}$ Cytochalasin isolated from Diplodia macrospora efficiently inhibited positioning of hypoxia-inducible factor- $1 \alpha$ at the VEGF promoter in ovarian cancer cells. ${ }^{30}$ Arthrinins isolated from Arthrinium spp. markedly inhibited VEGF receptor-2 in cancer cells. ${ }^{31}$

\section{Preliminary results for fungal products}

Citriquinone is a benzoquinone isolated from Penicillium citrinum and has notable activity against cancer cells. ${ }^{32}$ Chaetoglobosin, a secondary metabolite isolated from Penicillium aquamarinium, effectively induced cell cycle arrest and apoptosis in leukemia cells. It was also noted to exert inhibitory effects on cell migration and membrane ruffling. ${ }^{33}$
Diterpene alkaloids known as penitrems are also bioactive ingredients isolated from Penicillium with considerable anticancer activity against MCF-7 breast cancer cells. ${ }^{34}$ Fermented ginseng extracts isolated from Penicillium simplicissimum have activity against ES2 human clear-cell ovarian carcinoma cells. ${ }^{35}$ Eremophilane-type sesquiterpenes isolated from Penicillium spp. have activity against A-549 cancer cells. ${ }^{36}$ Dihydrothiophene-condensed chromone isolated from Penicillium oxalicum is effective against SW-620, A375, and HeLa cancer cells. ${ }^{37}$ There is a direct piece of evidence highlighting the autophagic cell death-inducing role of peneciraistin, a spiroketal molecule isolated from Penicillium raistrickii, in A549 lung cancer cells. Moreover, use of autophagy inhibitors induced apoptotic cell death in A549 cells. ${ }^{38}$ It is noteworthy that the mammosphere-forming ability of MCF-7 cells decreased significantly upon treatment with the 3-O-methylfunicone metabolite isolated from Penicillium pinophilum. ${ }^{39}$ Patulin isolated from Penicillium spp. has been shown to induce activation of caspase- 3 and to considerably enhance levels of phospho-EGR-1; indepth analysis revealed an EGR-1-mediated increase in expression of ATF3 in colorectal cancer cells. ${ }^{40}$

\section{MiRNA regulation}

miRNA is a non-coding RNA consisting of 21-22 nucleotides. The RNA polymerase binds to the promoter region of a specific DNA sequence and forms a hairpin structure of the pri-miRNA. The pri-miRNA is recognized by nuclear protein DGCR8. DGCR8 works synchronously with Drosha by cleaving nucleotides from hair pin of pri-miRNA to form pre-miRNA. This "pre-miRNA" transcript has the capacity to form a stem-loop structure, and is then processed into 21-22 nucleotide long miRNA by an enzyme called Dicer. Increasingly, it is being realized that miRNAs regulate other genes by binding to complementary sequences in the target gene. Perfect complementarity of binding leads to mRNA degradation of the target gene. Imperfect pairing inhibits translation of mRNA to protein. miRNAs have emerged as master regulators of the gene network.

Diaporine A (D261), a natural product of endophytic fungus, has been shown to effectively inhibit proliferation of non-small cell lung cancer cells by upregulating expression of miR-99a. It was shown mechanistically that miR-99a quantitatively controlled expression of mTOR. Diaporine A also induced regression of tumor growth in mice xenografted with NCI-H460 non-small cell lung cancer cells. ${ }^{41}$ Antrocin, a sesquiterpene lactone isolated from A. camphorata, a medicinal mushroom, considerably inhibited interleukin 
6-induced activation of STAT3, constitutive activation of STAT-3, and its nuclear accumulation. Let-7c was markedly increased in antrocin-treated cancer cells. More importantly, intraperitoneally administered antrocin induced regression of tumor growth in lung cancer tumor xenografts. ${ }^{42}$

Bostrycin, isolated from marine fungi, considerably upregulated miR-923 and miR-638 in A549 lung carcinoma cells. ${ }^{43}$ miR-638 is known to suppress progression of cancer, as evidenced by cell culture studies. Future studies should focus on identifying the potential protein network involved in cancer progression.

A. cinnamomea, a medicinal fungus, has considerable efficacy against SK-Hep-1 liver cancer cells. The MAPK and $\mathrm{PI} 3 \mathrm{~K} / \mathrm{Akt}$ signaling cascades were extensively reduced in SK-Hep-1 cells treated with extracts of $A$. cinnamomea. Surprisingly, the oncomirs miR-21 and miR-191, together with oncogenic clusters miR-106b-25 and miR-17-92, were effectively downregulated in SK-Hep-1 cells. ${ }^{44}$

Trametes robiniophila murris (Huaier) is a fungus with notable anticancer activity. Recently, it has been shown that Huaier stimulates expression of miR-26b-5p in A549 pulmonary adenocarcinoma cells. A549 cells transfected with miR-26b-5p displayed a higher apoptotic rate, and targeted inhibition of miR-26b-5p in A549 cells dramatically abrogated the Huaier-mediated anticancer effects. It was shown mechanistically that miR-26b-5p negatively regulated EZH2 in A549 cells. ${ }^{45}$

\section{Pharmacokinetic studies}

Aspergiolide (ASP)-A, an anthracycline isolated from a marine fungus, significantly inhibited tumor growth in $\mathrm{H} 22$ hepatoma-bearing mice. The distribution and pharmacokinetic parameters of ASP-A were studied by intraperitoneal injection of $15 \mathrm{mg} / \mathrm{kg}$ and $30 \mathrm{mg} / \mathrm{kg}$ of ASP-A at varying times. ASP-A achieved an effective concentration of drug and was noted to be broadly redistributed in vivo with a shorter half-life. ${ }^{46}$ Xyloketal B, isolated from Xylaria spp. (mangrove fungus), exerted inhibitory effects on cytochrome P450 (CYP)3A-mediated metabolism of midazolam in rats by markedly reducing hepatic CYP3A activity and expression. Xyloketal B may behave as a CYP3A4/Cyp3A2 substrate and/or inhibitor, which dramatically alters the pharmacokinetics of CYP3A4/Cyp3a2-mediated metabolism of coadministered drugs. ${ }^{47}$

\section{Clinical trials}

Schizophyllan (SPG) has previously been used in combination with conventional chemotherapy in a randomized controlled trial including 367 patients with inoperable recurrent gastric cancer, resulting in a considerable increase in median survival. SPG in combination with radiotherapy notably improved the time to recurrence and survival time in patients with stage II cervical cancer in this randomized controlled trial but not in those with stage III disease. ${ }^{48,49}$ In a prospective, randomized controlled trial involving 312 patients treated with surgery, chemotherapy (5-fluorouracil), radiotherapy, and SPG in various combinations, survival rates were notably longer in SPG-treated patients than in those who did not receive SPG. It is noteworthy that a markedly increased proportion of $\mathrm{CD}^{+}$cells $\left(\mathrm{HLA}-\mathrm{DR}^{+}\right.$) activation from total $\mathrm{CD}^{+}{ }^{+}$subset was evident with progressing disease. SPG-induced acceleration was observed in terms of recovery of activated $\mathrm{CD} 8^{+} \mathrm{T}$-cells in the $\mathrm{CD}^{+} \mathrm{T}$-cell subsets when compared with patients not treated with sizofiran (SPG) after radiotherapy. ${ }^{50} \mathrm{SPG}$ was also tested for efficacy in 15 patients with head and neck cancer, and the SPG-treated group had an $86.7 \%$ cumulative 5 -year survival rate. ${ }^{51}$

Basidiomycetes are a rich source of natural agents and a widely studied product is the protein-bound polysaccharide, PSK $\left(\right.$ Krestin $\left.^{\circledR}\right)$, with notable biological activity. There is a surprising piece of evidence suggesting that PSK-mediated anticancer effects were not significant either in early-stage cancers or in more advanced cases $(>\mathrm{S} 3$ or $>\mathrm{N} 3)$ in patients treated combinatorially with carbazilquinone + PSK. However, survival of patients whose disease was classified as S1 + S2(N1-2) was better on treatment with carbazilquinone + PSK ${ }^{52}$

In a randomized double-blind trial, PSK was given to colorectal cancer patients, and the results revealed improved disease-free survival $(P<0.05)$ and overall survival $(P<0.05)$ rates in the PSK group. Importantly, analysis of patients with polymorphonuclear leukocytes and undergoing treatment with PSK indicated notable biological activity, such as phagocytosis and chemotactic locomotion. ${ }^{53}$ There is also a direct piece of evidence suggesting that the 5-year disease-free survival rate was considerably high $(73.0 \%)$ in a group treated with chemotherapy + PSK when compared to that in patients treated with chemotherapy alone. Disease recurrence was reduced by $43.6 \%$ (95\% confidence interval [CI] 4.5-66) and mortality by $40.2 \%(95 \% \mathrm{CI}-12.5,68.3)$ in the PSK-treated group. Disease-free survival and overall survival in PSK-treated patients with stage III disease increased significantly, with a notable decrease in recurrence, particularly of lung metastases. ${ }^{54}$

Meta-analysis of three randomized controlled trials conducted in patients who had undergone curative resection also highlighted the effective role of PSK. A comparative analysis 
of chemotherapy alone and chemotherapy + PSK concluded that the disease-free survival risk ratio was $0.72(95 \%$ CI $0.58-0.90, P=0.003)$ and the overall survival risk ratio was 0.71 (95\% CI $0.55-0.90, P=0.006)$, thus signifying notable survival rates because of the effective synergy induced by the combinatorial approach. ${ }^{55}$

\section{Conclusion}

Rapidly developing resistance against a wide range of therapeutic interventions has added another layer of complexity and future studies must focus on identification of natural agents with clear anticancer activity. Fungal products have been shown to have considerable potential in delaying the progression of cancer and induction of apoptosis, as evidenced by in vitro and in vivo studies. It is becoming increasingly evident that fungal products can modulate the intracellular protein network, and a better understanding of the different cancers and their subtypes will be helpful in identification of most efficient bioactive ingredients will minimal off-target effects.

\section{Disclosure}

The authors report no conflicts of interest in this work.

\section{References}

1. Wang CL, Lu CY, Hsueh YC, Liu WH, Chen CJ. Activation of antitumor immune responses by Ganoderma formosanum polysaccharides in tumorbearing mice. Appl Microbiol Biotechnol. 2014;98(22):9389-9398.

2. Ko BS, Lu YJ, Yao WL, et al. Cordycepin regulates GSK-3 $\beta / \beta$-catenin signaling in human leukemia cells. PLoS One. 2013;8(9):e76320.

3. Song KS, Li G, Kim JS, et al. Protein-bound polysaccharide from Phellinus linteus inhibits tumor growth, invasion, and angiogenesis and alters Wnt/ $\beta$-catenin in SW480 human colon cancer cells. BMC Cancer. 2011;11:307.

4. Yeh CT, Rao YK, Ye M, et al. Preclinical evaluation of destruxin B as a novel Wnt signaling target suppressing proliferation and metastasis of colorectal cancer using non-invasive bioluminescence imaging. Toxicol Appl Pharmacol. 2012;261(1):31-41.

5. Park DK, Lim YH, Park HJ. Antrodia camphorata grown on germinated brown rice inhibits HT-29 human colon carcinoma proliferation through inducing G0/G1 phase arrest and apoptosis by targeting the $\beta$-catenin signaling. J Med Food. 2013;16(8):681-691.

6. Rudolph K, Serwe A, Erkel G. Inhibition of TGF- $\beta$ signaling by the fungal lactones (S)-curvularin, dehydrocurvularin, oxacyclododecindione and galiellalactone. Cytokine. 2013;61(1):285-296.

7. Serwe A, Anke T, Erkel G. The fungal secondary metabolite trichodimerol inhibits TGF- $\beta$ dependent cellular effects and tube formation of MDA-MB-231 cells. Invest New Drugs. 2009;27(6):491-502.

8. Jeong MH, Lee CM, Lee SW, et al. Cordycepin-enriched Cordyceps militaris induces immunomodulation and tumor growth delay in mousederived breast cancer. Oncol Rep. 2013;30(4):1996-2002.

9. $\mathrm{Su}$ J, Zhao P, Kong L, et al. Trichothecin induces cell death in NF- $\kappa B$ constitutively activated human cancer cells via inhibition of IKK $\beta$ phosphorylation. PLoS One. 2013;8(8):e71333.

10. Li YX, Himaya SW, Dewapriya P, Zhang C, Kim SK. Fumigaclavine C from a marine-derived fungus Aspergillus fumigatus induces apoptosis in MCF-7 breast cancer cells. Mar Drugs. 2013;11(12):5063-5086.
11. Deng Q, Yu X, Xiao L, et al. Neoalbaconol induces energy depletion and multiple cell death in cancer cells by targeting PDK1-PI3-K/Akt signaling pathway. Cell Death Dis. 2013;4:e804.

12. Xu W, Huang JJ, Cheung PC. Extract of Pleurotus pulmonarius suppresses liver cancer development and progression through inhibition of VEGF-induced PI3K/AKT signaling pathway. PLoS One. 2012; 7(3):e34406.

13. Ozcan Z, Gül G, Yaman I. Ochratoxin A activates opposing c-MET/ PI3K/Akt and MAPK/ERK 1-2 pathways in human proximal tubule HK-2 cells. Arch Toxicol. July 8, 2014. [Epub ahead of print].

14. Anninou N, Chatzaki E, Papachristou F, et al. Mycotoxins' activity at toxic and sub-toxic concentrations: differential cytotoxic and genotoxic effects of single and combined administration of sterigmatocystin, ochratoxin A and citrinin on the hepatocellular cancer cell line Hep3B. Int J Environ Res Public Health. 2014;11(2):1855-1872.

15. Buommino E, Paoletti I, De Filippis A, et al. 3-O-Methylfunicone, a metabolite produced by Penicillium pinophilum, modulates ERK1/2 activity, affecting cell motility of human mesothelioma cells. Cell Prolif. 2010;43(2):114-123.

16. Chen YY, Chou PY, Chien YC, Wu CH, Wu TS, Sheu MJ. Ethanol extracts of fruiting bodies of Antrodia cinnamomea exhibit anti-migration action in human adenocarcinoma CL1-0 cells through the MAPK and PI3K/AKT signaling pathways. Phytomedicine. 2012;19(8-9):768-778.

17. Hsiao CJ, Hsiao G, Chen WL, et al. Cephalochromin induces G0/G1 cell cycle arrest and apoptosis in A549 human non-small-cell lung cancer cells by inflicting mitochondrial disruption. J Nat Prod. 2014; 77(4):758-765.

18. Kang YJ, Kim IY, Kim EH, et al. Paxilline enhances TRAIL-mediated apoptosis of glioma cells via modulation of c-FLIP, survivin and DR5. Exp Mol Med. 2011;43(1):24-34.

19. Kim YJ, Park HB, Yoo JH, Kwon HC, Kim J, Yang HO. Glionitrin A, a new diketopiperazine disulfide, activates ATM-ATR-Chk1/2 via 53BP1 phosphorylation in DU145 cells and shows antitumor effect in xenograft model. Biol Pharm Bull. 2014;37(3):378-386.

20. Palanivel K, Kanimozhi V, Kadalmani B, Akbarsha MA. Verrucarin A, a protein synthesis inhibitor, induces growth inhibition and apoptosis in breast cancer cell lines MDA-MB-231 and T47D. Biotechnol Lett. 2013;35(9):1395-1403.

21. Miao S, Mao X, Pei R, et al. Lepista sordida polysaccharide induces apoptosis of Hep-2 cancer cells via mitochondrial pathway. Int J Biol Macromol. 2013;61:97-101.

22. Zhang S, Nie S, Huang D, Huang J, Wang Y, Xie M. Polysaccharide from Ganoderma atrum evokes antitumor activity via Toll-like receptor 4-mediated NF- $\mathrm{BB}$ and mitogen-activated protein kinase signaling pathways. J Agric Food Chem. 2013;61(15):3676-3682.

23. Zhang S, Nie S, Huang D, Li W, Xie M. Immunomodulatory effect of Ganoderma atrum polysaccharide on CT26 tumor-bearing mice. Food Chem. 2013;136(3-4):1213-1219.

24. Suarez-Arroyo IJ, Rosario-Acevedo R, Aguilar-Perez A, et al. Antitumor effects of Ganoderma lucidum (reishi) in inflammatory breast cancer in in vivo and in vitro models. PLoS One. 2013;8(2):e57431.

25. Chen RJ, Hung CM, Chen YL, Wu MD, Yuan GF, Wang YJ. Monascuspiloin induces apoptosis and autophagic cell death in human prostate cancer cells via the Akt and AMPK signaling pathways. J Agric Food Chem. 2012;60(29):7185-7193.

26. Rao YK, Wu AT, Geethangili M, et al. Identification of antrocin from Antrodia camphorata as a selective and novel class of small molecule inhibitor of Akt/mTOR signaling in metastatic breast cancer MDAMB-231 cells. Chem Res Toxicol. 2011;24(2):238-245.

27. Li N, YiZ, Wang Y, et al. Differential proteomic analysis of HL60 cells treated with secalonic acid $F$ reveals caspase 3-induced cleavage of Rho GDP dissociation inhibitor 2. Oncol Rep. 2012;28(6):2016-2022.

28. Chan YY, Chang CS, Chien LH, Wu TF. Apoptotic effects of a high performance liquid chromatography (HPLC) fraction of Antrodia camphorata mycelia are mediated by down-regulation of the expressions of four tumor-related genes in human non-small cell lung carcinoma A549 cell. J Ethnopharmacol. 2010;127(3):652-661. 
29. García-Caballero M, Marí-Beffa M, Cañedo L, et al. Toluquinol, a marine fungus metabolite, is a new angiosuppressor that interferes with the Akt pathway. Biochem Pharmacol. 2013;85(12):1727-1740.

30. Luo H, Li B, Li Z, Cutler SJ, Rankin GO, Chen YC. Chaetoglobosin K inhibits tumor angiogenesis through downregulation of vascular epithelial growth factor-binding hypoxia-inducible factor $1 \alpha$. Anticancer Drugs. 2013;24(7):715-724.

31. Ebada SS, Schulz B, Wray V, et al. Arthrinins A-D: novel diterpenoids and further constituents from the sponge derived fungus Arthrinium sp. Bioorg Med Chem. 2011;19(15):4644-4651.

32. Ranji PK, Wijeyaratne SC, Jayawardana KH, Gunaherath GM. Citriquinones $\mathrm{A}$ and $\mathrm{B}$, new benzoquinones from Penicillium citrinum. Nat Prod Commun. 2013;8(10):1431-1434.

33. Knudsen PB, Hanna B, Ohl S, et al. Chaetoglobosin A preferentially induces apoptosis in chronic lymphocytic leukemia cells by targeting the cytoskeleton. Leukemia. 2014;28(6):1289-1298.

34. Sallam AA, Houssen WE, Gissendanner CR, Orabi KY, Foudah AI, El Sayed KA. Bioguided discovery and pharmacophore modeling of the mycotoxic indole diterpene alkaloids penitrems as breast cancer proliferation, migration, and invasion inhibitors. Medchemcomm. 2013;4(10).

35. Fu Y, Yin Z, Wu L, Yin C. Fermentation of ginseng extracts by Penicillium simplicissimum GS33 and anti-ovarian cancer activity of fermented products. World J Microbiol Biotechnol. 2014;30(3):1019-1025.

36. Lin A, Wu G, Gu Q, Zhu T, Li D. New eremophilane-type sesquiterpenes from an Antarctic deep sea derived fungus, Penicillium sp. PR19 N-1. Arch Pharm Res. 2014;37(7):839-844.

37. Sun YL, Bao J, Liu KS, et al. Cytotoxic dihydrothiophene-condensed chromones from the marine-derived fungus Penicillium oxalicum. Planta Med. 2013;79(15):1474-1479.

38. Pan X, Liu D, Wang J, et al. Peneciraistin C induces caspase-independent autophagic cell death through mitochondrial-derived reactive oxygen species production in lung cancer cells. Cancer Sci. 2013; 104(11):1476-1482.

39. Buommino E, Tirino V, De Filippis A, et al. 3-O-methylfunicone, from Penicillium pinophilum, is a selective inhibitor of breast cancer stem cells. Cell Prolif. 2011;44(5):401-409.

40. Kwon O, Soung NK, Thimmegowda NR, et al. Patulin induces colorectal cancer cells apoptosis through EGR-1 dependent ATF3 up-regulation. Cell Signal. 2012;24(4):943-950.

41. Song Y, Dou H, Wang P, et al. A novel small-molecule compound diaporine A inhibits non-small cell lung cancer growth by regulating miR-99a/mTOR signaling. Cancer Biol Ther. 2014;15(10):1423-1430.

42. Yeh CT, Huang WC, Rao YK, et al. A sesquiterpene lactone antrocin from Antrodia camphorata negatively modulates JAK2/STAT3 signaling via microRNA let-7c and induces apoptosis in lung cancer cells. Carcinogenesis. 2013;34(12):2918-2928.
43. Chen WS, Hou JN, Guo YB, et al. Bostrycin inhibits proliferation of human lung carcinoma A549 cells via downregulation of the PI3K/Akt pathway. J Exp Clin Cancer Res. 2011;30:17.

44. Chen YJ, Thang MW, Chan YT, et al. Global assessment of Antrodia cinnamomea-induced microRNA alterations in hepatocarcinoma cells. PLoS One. 2013;8(12):e82751.

45. Wu T, Chen W, Liu S, et al. Huaier suppresses proliferation and induces apoptosis in human pulmonary cancer cells via upregulation of miR26b-5p. FEBS Lett. 2014;588(12):2107-2114.

46. Wang Y, Qi X, Li D, Zhu T, Mo X, Li J. Anticancer efficacy and absorption, distribution, metabolism, and toxicity studies of aspergiolide A in early drug development. Drug Des Devel Ther. 2014;8:1965-1977.

47. Su JH, Chang C, Xiang Q, et al. Xyloketal B, a marine compound, acts on a network of molecular proteins and regulates the activity and expression of rat cytochrome P450 3a: a bioinformatic and animal study. Drug Des Devel Ther. 2014;8:2555-2602.

48. Okamura K, Suzuki M, Chihara T, et al. Clinical evaluation of schizophyllan combined with irradiation in patients with cervical cancer. A randomized controlled study. Cancer. 1986;58(4):865-872.

49. Okamura K, Hamazaki Y, Yajima A, Noda K. Adjuvant immunotherapy: two randomized controlled studies of patients with cervical cancer. Biomed Pharmacother. 1989;43(3):177-181.

50. Miyazaki K, Mizutani H, Katabuchi H, Fukuma K, Fujisaki S, Okamura H. Activated (HLA-DR+) T-lymphocyte subsets in cervical carcinoma and effects of radiotherapy and immunotherapy with sizofiran on cell-mediated immunity and survival. Gynecol Oncol. 1995; 56(3):412-420.

51. Kimura Y, Tojima H, Fukase S, Takeda K. Clinical evaluation of sizofilan as assistant immunotherapy in treatment of head and neck cancer. Acta Otolaryngol Suppl. 1994;511:192-195.

52. Kondo T, Sakamoto J, Nakazato H. Alternating immunochemotherapy of advanced gastric carcinoma: a randomized comparison of carbazilquinone and PSK to carbazilquinone in patients with curative gastric resection. Biotherapy. 1991;3(4):287-295.

53. Torisu M, Hayashi Y, Ishimitsu T, et al. Significant prolongation of disease-free period gained by oral polysaccharide K (PSK) administration after curative surgical operation of colorectal cancer. Cancer Immunol Immunother. 1990;31(5):261-268.

54. Ohwada S, Ikeya T, Yokomori T, et al. Adjuvant immunochemotherapy with oral tegafur/uracil plus PSK in patients with stage II or III colorectal cancer: a randomised controlled study. Br J Cancer. 2004;90(5): 1003-1010.

55. Sakamoto J, Morita S, Oba K, et al; Meta-Analysis Group of the Japanese Society for Cancer of the Colon Rectum. Efficacy of adjuvant immunochemotherapy with polysaccharide $\mathrm{K}$ for patients with curatively resected colorectal cancer: a meta-analysis of centrally randomized controlled clinical trials. Cancer Immunol Immunother. 2006;55(4):404-411.
Drug Design, Development and Therapy

\section{Publish your work in this journal}

Drug Design, Development and Therapy is an international, peerreviewed open-access journal that spans the spectrum of drug design and development through to clinical applications. Clinical outcomes, patient safety, and programs for the development and effective, safe, and sustained use of medicines are a feature of the journal, which

\section{Dovepress}

has also been accepted for indexing on PubMed Central. The manuscript management system is completely online and includes a very quick and fair peer-review system, which is all easy to use. Visit http://www.dovepress.com/testimonials.php to read real quotes from published authors. 